Typeset with jpsj2.cls $<$ ver.1.2 $>$

Full PAPER

\title{
Quasi-one-dimensional spin-density-wave states in the presence of two commensurate potentials and an interchain misfit
}

\author{
T. ITAKURA * \\ Department of Physics, Kyoto-University, Kyoto 606-8502, Japan \\ TEL:075-753-3750
}

(Received November 16, 2018)

\begin{abstract}
Spin density wave (SDW) states of a quasi-one-dimensional system with an incommensurate wave vector perpendicular to the chain have been studied in the presence of two kinds of commensurate potentials, which originate in a quarter-filled band and dimerization along the chain. In terms of a phase variable of the SDW order parameter, we treat classically the two-dimensional Hamiltonian, which includes both acoustic excitations with long wave length and a vortex excitation with short wave length. A phase diagram on the plane of temperature and chemical potential (where the latter corresponds to the deviation of the transverse wave vector from the commensurate one) exhibits a variety of states given by the commensurate SDW state without charge density, the commensurate SDW state with charge density, the incommensurate SDW state and the disordered state.
\end{abstract}

KEYWORDS: spin density wave, phase, quarter-filled band, commensurate-incommensurate transition, renormalization group method, vortex, dimerization

\section{Introduction}

In several kinds of density wave systems, a commensurate-incommensurate transition has been studied theoretically by varying a misfit from the commensurate wave vector (or a chemical potential). Such a transition is followed by a formation of the soliton of the phase of density wave, since the energy gain of a commensurability competes with the increase of elastic energy driven by the misfit. Pokrovsky and Talapov have first studied such a phenomenon in a two-dimensional classical system by using a renormalization group method. ${ }^{1}$ Such a method has been applied to the sine-Gordon model ${ }^{2,3}$ and the Coulomb gas model. ${ }^{4}$

In contrast to the one-dimensional case, it is crucial to take into account the effect of a vortex in the two-dimensional case, which appears at finite temperatures due to a thermal excitation. ${ }^{5}$ The vortex destroys the quasi-long range order resulting in the disordered phase. The phase diagram of commensurate and incommensurate states in the presence of the vortex interaction has been studied by using the renormalization group method (for $\mu=0)^{6,7}$ and the exact result (for finite $\mu$ ), ${ }^{8,9}$ where $\mu$ is the chemical potential corresponding to the misfit. The commensurability potential is characterized by the $p$-fold periodic potential with respect

${ }^{*}$ E-mail address: toshifumiitakura@scphys.kyoto-u.ac.jp 
to the phase. The case for $p=2$ has been studied extensively by applying the renormalization group method to a one-dimensional quantum system, ${ }^{10}$ in which the vortex and the misfit correspond to the anisotropic interaction of the spin freedom and an external magnetic field, respectively.

These studies have been devoted to the system only with a single commensurate potential while that with two kinds of commensurate potentials is of interest to investigate the following quasi-one-dimensional conductors, Bechgaard salts. ${ }^{11-13}$ The salts, which have a dimerized and quarter-filled conduction band, exhibit the spin density wave (SDW) state at low temperatures and undergoes a commensurate-incommensurate transition perpendicular to chain under effective pressures. ${ }^{14}$ The interchain interaction including the misfit of the phase difference between chains competes with intrachain commensurability energies where the misfit originates in the nesting of Fermi surface ${ }^{15}$ and/or exchange energy due to the various kinds of interchain hopping. ${ }^{4,16}$ When the repulsive interaction for the nearest-neighbor sites is large, the commensurability energy at quarter-filling leads to the coexistence of SDW with the charge ordering. ${ }^{17,18}$ However, the dimerization leads to an effectively half-filled band and is in favor of pure SDW state (i.e., the absence of charge ordering). In terms of a transfer integral method, the role of interchain misfit in the system with these two kinds of potentials has been examined for two-coupled chains ${ }^{19}$ and for a quasi-one-dimensional system, where the phase diagram for the latter model has been obtained on the plane of an intrachain misfit and temperature. ${ }^{20}$

In the present study, we examine the effect of these two commensurate energies on the quasi-one-dimensional quarter-filled SDW system with a transverse misfit by adding dimerization and vortex to the previous model. ${ }^{4}$ In $\S 2$, the classical two dimensional Hamiltonian for SDW state is derived by expanding the interchain coupling up to the second order with respect to phase difference between chains. The renormalization group equations are derived. In $\S 3$, we obtain the phase diagram of the commensurate SDW state and the incommensurate SDW state and the disordered state (induced by the vortex). In $\S 4$, discussion is given.

\section{Formulation}

We consider the SDW state of the quasi-one-dimensional electron system with the mean-

field order parameter given by $\Delta_{2 k_{\mathrm{F}}}^{i} \cos \left(2 k_{\mathrm{F}} x_{0}+\theta_{i}\right)$ where $\Delta_{2 k_{\mathrm{F}}}^{i}$ and $\theta_{i}$ denote the amplitude and the phase of the $i$-th chain, respectively. The effective Hamiltonian describing the charge fluctuation in the classical case is written as

$$
\begin{gathered}
\mathcal{H}_{\mathrm{Q} 1 \mathrm{D}}=\sum_{i} \int \mathrm{d} x_{0}\left[A\left(\frac{\mathrm{d} \theta_{i}\left(x_{0}\right)}{\mathrm{d} x_{0}}\right)^{2}-W_{c} \cos 4 \theta_{i}\left(x_{0}\right)\right. \\
\left.+J_{0} \cos \left(\theta_{i}\left(x_{0}\right)-\theta_{i+1}\left(x_{0}\right)+\mu\right)\right],
\end{gathered}
$$


where the $A$-term is an elastic energy while the $W_{c^{-}}$and $W_{d^{-}}$terms correspond to the commensurability energy at quarter-filling and that with dimerization, respectively. Equation (1) for two-chain has been studied previously. ${ }^{19}$ In the case of $W_{d}=J_{0}=0$, the spatial variation with a periodic array of $(\uparrow, 0, \downarrow, 0)$ is obtained for $W_{c}>0$, which is found for large nearest-neighbor repulsion. ${ }^{17,18}$ The $J_{0}$-term represents the interchain interaction with $\mu$ being a misfit parameter corresponding to the phase difference between nearest-neighbor chains. We examine only the case of $J_{0}<0$, because the replacement of $\theta_{i} \rightarrow \theta_{i}+\pi$ in the even chain leads to the same result as that of $J_{0}>0$. Since the calculation of eq. (1) is very complicated, we replace it by an effective one, which consists of acoustic excitation with long wave length and the excitation with short wave length. The former, which leads to the anisotropic twodimensional SDW state at low temperatures, is obtained by expanding the $J_{0}$-term in eq. (1) with respect to $\theta_{i}\left(x_{0}\right)-\theta_{i+1}\left(x_{0}\right)+\mu$ up to the second order. The continuum limit is taken as $\theta_{i}\left(x_{0}\right) \rightarrow \theta\left(x_{0}, y_{0}\right)$ with $y_{0}=i b$ where $b$ is the lattice constant for interchain direction. The latter, which corresponds to the higher order terms and is dominant at high temperatures near the critical temperature, can be represented in terms of the vortex field, ${ }^{6,7}$ which is relevant to the potential for $\theta$ with a periodicity, $2 \pi$. The potential for the vortex field is expressed as

$$
W_{v} \cos 2 \pi K \phi(x, y)
$$

which resembles a one-dimensional quantum system (see Appendix). In eq.(2), $\phi$ is the conjugate phase variable defined as $\partial \theta / \partial x_{0}=i \beta \partial \phi / \partial y_{0}$ and $\partial \theta / \partial y_{0}=-i \beta^{-1} \partial \phi / \partial x_{0}$ with $\beta=b\left(\left|J_{0}\right| / 2 A\right)^{1 / 2}$. The quantity $K$ is given by $K \equiv \sqrt{2 A\left|J_{0}\right|} / T_{0}$, and the quantity $W_{v}$ is the coupling constant for the vortex field whose form depends on a microscopic model. The quantity $T_{0}$ is the temperature. By use of dimensionless quantities,

$$
x=\sqrt{\frac{W_{c}}{A}} x_{0}, y=\frac{y_{0}}{b}, T=\frac{T_{0}}{\sqrt{A W_{c}}}, \quad J=\frac{\left|J_{0}\right|}{W_{c}},
$$

the effective Hamiltonian for eq.(1) is given by ${ }^{4}$

$$
\begin{aligned}
\frac{\mathcal{H}_{\mathrm{Q} 1 \mathrm{D}}}{T_{0}}= & \iint \mathrm{d} x \mathrm{~d} y \\
\times & {\left[\frac{1}{T}\left(\frac{\partial \theta(x, y)}{\partial x}\right)^{2}+\frac{J}{2 T}\left(\frac{\partial \theta(x, y)}{\partial y}-\mu\right)^{2}\right.} \\
& -\frac{1}{T} \cos 4 \theta(x, y)-\frac{1}{T}\left(\frac{W_{d}}{W_{c}}\right) \sin 2 \theta(x, y) \\
& \left.-\frac{1}{T}\left(\frac{W_{v}}{W_{c}}\right) \cos 2 \pi K \phi(x, y)\right] .
\end{aligned}
$$

For the convenience of applying a renormalization group method, eq. (3) is rewritten as

$$
\begin{aligned}
\frac{\mathcal{H}_{\mathrm{Q} 1 \mathrm{D}}}{T_{0}} & =\iint \mathrm{d} x \mathrm{~d} y \\
& \times\left[\frac{K}{2 \beta}\left(\frac{\partial \theta(x, y)}{\partial x}\right)^{2}+\frac{K \beta}{2}\left(\frac{\partial \theta(x, y)}{\partial y}-\mu\right)^{2}\right.
\end{aligned}
$$


J. Phys. Soc. Jpn.

Full PAPER

$$
\begin{aligned}
& -2 y_{4} \cos 4 \theta(x, y)-2 y_{2} \sin 2 \theta(x, y) \\
& \left.-2 y_{v} \cos 2 \pi K \phi(x, y)\right],
\end{aligned}
$$

where $K=\sqrt{2 J} / T, \beta \equiv \sqrt{J / 2}, y_{4}=1 / 2 T, y_{2} \equiv\left(W_{d} / W_{c}\right) / 2 T$ and $y_{v} \equiv\left(W_{v} / W_{c}\right) / 2 T$. The renormalization group equations are calculated by use of Coulomb gas method while the same results are obtained by the use of the method of Giamarchi and Schulz. ${ }^{10}$ These equations are obtained as

$$
\begin{aligned}
\frac{\mathrm{d}}{\mathrm{d} l} \frac{1}{K} & =-\frac{4 \pi \bar{y}_{2}^{2}}{K^{2}} J_{0}(2 \bar{\mu})-\frac{16 \pi \bar{y}_{4}^{2}}{K^{2}} J_{0}(4 \bar{\mu})+4 \pi^{3} \bar{y}_{v}^{2}, \\
\frac{\mathrm{d} \bar{y}_{2}}{\mathrm{~d} l} & =\left(2-\frac{1}{\pi K}\right) \bar{y}_{2}-2 \pi \bar{y}_{2} \bar{y}_{4}, \\
\frac{\mathrm{d} \bar{y}_{4}}{\mathrm{~d} l} & =\left(2-\frac{4}{\pi K}\right) \bar{y}_{4}-\pi \bar{y}_{2}^{2}, \\
\frac{\mathrm{d} \bar{y}_{v}}{\mathrm{~d} l} & =(2-\pi K) \bar{y}_{v}, \\
\frac{\mathrm{d} \bar{\mu}}{\mathrm{d} l}=\bar{\mu} & -\frac{4 \pi \bar{y}_{2}^{2}}{K} J_{1}(2 \bar{\mu})-\frac{8 \pi \bar{y}_{4}^{2}}{K} J_{1}(4 \bar{\mu}), \\
\frac{\mathrm{d} \ln \beta}{\mathrm{d} l} & =\frac{4 \pi \bar{y}_{2}^{2}}{K} J_{2}(2 \bar{\mu})+\frac{16 \pi \bar{y}_{4}^{2}}{K} J_{2}(4 \bar{\mu})
\end{aligned}
$$

where $\overline{y_{4}} \equiv \beta \alpha^{2} y_{4}, \overline{y_{2}} \equiv \beta \alpha^{2} y_{2}, \overline{y_{v}} \equiv \beta \alpha^{2} y_{v}$ and $\bar{\mu} \equiv \beta \alpha \mu$. The quantity $\alpha$ is short-length cutoff of the order of lattice constant where the constant is taken as unity. We note that the second order corrections to eqs. (6) and (7) are obtained by examining the response function up to third order, $y_{2}^{2} y_{4} \cdot{ }^{21,22}$

\section{Phase Diagram}

The renormalization group equations (eqs. (5)-(9)) are calculated numerically by choosing initial conditions as $\left.1 / K\left(=T /(2 J)^{1 / 2}\right), \bar{\mu}\left(=\alpha \mu(J / 2)^{1 / 2}\right)\right), y_{4}=\alpha^{2} K / 4$ and $y_{2}=\left(W_{d} / W_{c}\right) y_{4}$. The vortex interaction term is taken as $\bar{y}_{v}=\exp \left(-\pi^{2} K / 2\right)$ as found in the isotopic XY model. ${ }^{6}$ In the present calculation, we take $\alpha=0.5$ and use an approximation for the Bessel function such that $J_{0}(z)=1, J_{1}(z)=z / 2$ for $z \lesssim 1.8$ and zero otherwise. These are possible choices to reproduce the well known result for $y_{2}=0$ (see Fig. 1).

From the solution of the renormalization equations, we obtain the following states. (i)The irrelevant $\bar{\mu}$ leads to two kinds of commensurate states. The relevant $y_{2}$ corresponds to the commensurate state of pure SDW ( $\mathrm{C}_{\mathrm{I}}$-phase) while the irrelevant $\overline{y_{2}}$ and relevant $\overline{y_{4}}$ correspond to the commensurate SDW state coexisting with charge order ( $\mathrm{C}_{\mathrm{II}}$-phase) (ii) When $\bar{\mu}$ is relevant, the incommensurate state (IC-phase) is obtained for the irrelevant $\overline{y_{v}}$ and the disordered state with the vortex (disorder-phase) is obtained for the relevant $\overline{y_{v}}$. These fixed points are summarized in Table I where $K^{*}$ denotes a finite value.

First, we examine the case that the dimerization is absent $\left(y_{2}=0\right)$. In the present calculation, we take $\alpha=0.5$ and use an approximation for the Bessel function such that $J_{0}(z)=1$, 


\begin{tabular}{cccccc}
\hline & $1 / K$ & $\overline{y_{2}}$ & $\overline{y_{4}}$ & $\overline{y_{v}}$ & $\bar{\mu}$ \\
\hline $\mathrm{C}_{\mathrm{I}}$ & 0 & $\infty$ & $-\infty$ & 0 & 0 \\
$\mathrm{C}_{\mathrm{II}}$ & 0 & 0 & $\infty$ & 0 & 0 \\
$\mathrm{IC}$ & $1 / K^{*}$ & $(0)$ & $(0)$ & 0 & $\infty$ \\
Disorder & $\infty$ & 0 & 0 & $\infty$ & $\infty$ \\
\hline
\end{tabular}

Table I. Fixed point values for respective phases. The parenthesis indicates the expected value.

$J_{1}(z)=z / 2$ for $z \lesssim 1.8$ and zero otherwise. These are possible choices, which reproduce well the previous results. ${ }^{8}{ }^{9}$ For small $\bar{\mu}$, the smaller $\alpha$ enhances the IC region while the larger $\alpha$ diminishes the IC region In Fig. 1, there are three fixed points corresponding to $\mathrm{C}_{\mathrm{II}}$, IC and disorder phases (Fig. 1). In the $\mathrm{C}_{\mathrm{II}}$-phase, the charge density becomes long range while, in the IC-phase, the gapless excitation due to a finite $K^{*}(=K(\infty))$ results in the power low decay of the response function of density waves. ${ }^{4}$ In the disorder phase, the vortex, which is activated by thermal fluctuations, destroys the (quasi-)long range order and the response function for the density wave decays exponentially. There exists the incommensurate state between the commensurate state and the disordered state if $\bar{\mu} \neq 0$. The absence of the incommensurate state for $\bar{\mu}=0$ is understood as follows. Equations (7) and (8) shows the irrelevant $\overline{y_{4}}\left(\overline{y_{v}}\right)$ for $1 / K^{*}>\pi / 2\left(1 / K^{*}<\pi / 2\right)$ so that either $\overline{y_{4}}$ or $\overline{y_{v}}$ is relevant for $\bar{\mu}=0$. We remark followings for other boundaries. Equation (8) shows that $1 / K^{*}$ is $\pi / 2$ at the boundary between disorder phase and IC-phase. The boundary between $\mathrm{C}_{\mathrm{II}}$ and IC at $\mathrm{T}=0$ (i.e., $1 / K=0$ ) is given by $\beta \mu=2 \sqrt{2} / \pi$, which can be derived analytically by examining the condition for the formation of the soliton. For $\beta \mu>2 \sqrt{2} / \pi, \overline{y_{4}}$ is always irrelevant, and the critical temperature for $\overline{y_{v}}(l) \rightarrow 0$ is almost independent of $\overline{y_{4}}$. The boundary at $1 / K=0$ is obtained from eqs. (6) and (8), which lead to $\mathrm{d}\left({\overline{y_{4}}}^{2}-2{\overline{y_{2}}}^{2}\right) / \mathrm{d} l=4\left({\overline{y_{4}}}^{2}-2{\overline{y_{2}}}^{2}\right)$. When temperature is decreased (i.e., $\bar{y}_{4}$ is increased) with fixed $y_{2} / y_{4}=W_{d} / W_{c}$, it turns out that the $\mathrm{C}_{\mathrm{I}}$ phase moves into the $\mathrm{C}_{\mathrm{II}}$ phase for $y_{2}<\sqrt{2} y_{4}$ corresponding to the small dimerization. With increasing $1 / K$, the region of $\mathrm{C}_{\mathrm{I}}$-phase increases, because the effect of $1 / K$ on the scaling dimension for $\overline{y_{4}}$ (i.e., $2-4 / \pi K)$ is larger than that for $\overline{y_{2}}$ (i.e., $2-1 / \pi K$ ). In addition, the high temperature corresponds to large $1 / K$ due to $1 / K=T / \sqrt{2 J}$ so that $\mathrm{C}_{\text {I }}$ phase $\left(\mathrm{C}_{\text {II }}\right.$ phase $)$ is obtained at high (low) temperatures. With increasing $1 / K$ from the $\mathrm{C}_{\mathrm{II}}$-phase which is obtained for $1 / K^{*}<\pi / 2$, one always finds the $\mathrm{C}_{\mathrm{I}}$-phase, which is located in $\pi / 2<1 / K^{*}<2 \pi$.

Next we examine the state in the presence of both dimerization and quarter-filled commensurability energy, which exhibit four fixed points as shown in Table 1. Their states are given by two kinds of commensurate states. $\left(\mathrm{C}_{\mathrm{I}}\right.$-phase and $\mathrm{C}_{\mathrm{II}}$-phase $)$, incommensurate state (IC-phase) and disordered state (disorder-phase). In Fig.3, the phase diagram is shown on the plane of $\beta \mu$ and $1 / K$ where $y_{2} / y_{4}=W_{d} / W_{c}=0.5$. The $\mathrm{C}_{\mathrm{I}}$-phase (the commensurate state of the pure $2 k_{F}$ SDW state) is found at high temperatures and for $\beta \mu$ smaller than a criti- 
cal value. The $\mathrm{C}_{\mathrm{II}}$-phase (the commensurate state coexisting with $4 k_{F} \mathrm{CDW}$ state) is found at low temperatures and the critical value of $\beta \mu$ is larger than that of $\mathrm{C}_{\mathrm{I}}$-phase. Compared with Fig. 1, it turns out that dimerization has an effect of producing the region of $\mathrm{C}_{\mathrm{I}}$-phase around the triple point in Fig. 1, at which disorder, IC and $\mathrm{C}_{\mathrm{II}}$ merge together. Note that the boundary between $\mathrm{C}_{\mathrm{II}}$ and IC at $T(=1 / K)=0$ does not change within the visible scale even in the presence of both $y_{2}$ and $y_{v}$, which become irrelevant at low temperatures. With decreasing temperature with fixed $\beta \mu$, one obtains following four kinds of successive transitions: (I) disorder $\rightarrow \mathrm{C}_{\mathrm{I}} \rightarrow \mathrm{C}_{\mathrm{II}}$, (II) disorder $\rightarrow \mathrm{C}_{\mathrm{I}} \rightarrow \mathrm{IC} \rightarrow \mathrm{C}_{\mathrm{II}}$, (III) disorder $\rightarrow$ IC $\rightarrow \mathrm{C}_{\mathrm{II}}$, and $(\mathrm{IV})$ disorder $\rightarrow$ IC. However the tricritical point for $\mathrm{C}_{\mathrm{I}} \mathrm{C}_{\mathrm{II}}$ and IC depends on the choice of $\alpha$ which leads to another transition of disorder $\rightarrow$ IC $\rightarrow \mathrm{C}_{\mathrm{I}} \rightarrow \mathrm{C}_{\mathrm{II}}$ instead of (II). Therefore the analysis of such a detail is beyond the present scheme of the renormalization group equation. The remained three kinds of transition seem to be reasonable as the result of combining the commensurability of $p=2$ and that of $p=4$.

\section{Discussion}

We examined the effect of transverse misfit (chemical potential) on the quasi-onedimensional SDW system which includes two-kinds of commensurate potentials coming from the dimerized quarter-filled band. The anisotropic two-dimensional SDW system is treated classically since the quantum fluctuations may have an effect of renormalizing the amplitude of $W_{c}$ and $W_{d}$ but does not change qualitatively the form of the potential. We found the phase diagram, which exhibits a variety of states, on the plane of chemical potential and temperature when the two kinds of commensurate potentials compete with each other. The transition from the disordered state to the incommensurate SDW state is the Kosterlitz-Thouless transition. It is of interest whether such a transition in addition to commensurate-incommensurate transition is related to the sub-phases of (TMTSF) ${ }_{2} \mathrm{PF}_{6}$ salt. ${ }^{26}$

Finally we note the phase diagram of Fig. 3, which is the result obtained in the classical quasi-one-dimensional system with two-kinds of periodic potential competing each other, anisotropic misfit parameter, and vortex interaction induced by interchain coupling. This result in two-dimensional classical case has a common feature with the previous one, ${ }^{20}$ (except for the state around the triple point) in which the interchain coupling is treated in the mean-field theory and the transfer integral method is applied to treat the thermal fluctuation exactly. In these cases, two kinds of potentials are the same but the misfit in the previous case is in the chain and the present case is perpendicular to the chain. Compared with the phase diagram (Fig. 8 in the previous case ${ }^{20}$ ), we found the similarity in the sense that $\mathrm{C}_{\mathrm{I}}\left(\mathrm{C}_{1}\right)$ is the pure SDW state, $\mathrm{C}_{\mathrm{II}}\left(\mathrm{C}_{2}\right)$ is the SDW state coexisting with the $\mathrm{CDW}$ and the disorder phase denotes the absence of the long range order, (i.e., $\langle\cos \theta\rangle=0$ ). The notable difference is between the incommensurate along the chain and that perpendicular to chain. It is considered that such a nice correspondence between these two results may be attributable to the proper 
treatment of the thermal fluctuation.

\section{Acknowledgements}

This work is supported by the Grant-in-Aid for the 21st Century COE "Center for Diversity and Universality in Physics" from the Ministry of Education, Culture, Sports, Science and Technology (MEXT) of Japan.

\section{Appendix: Classical 2D Hamiltonian vs. quantum 1D Hamiltonian}

Using the path integral methods, we show that the quantum one-dimensional (1D model is equivalent to the present classical two-dimensional (2D) model. Using the StratonovichHubbard identity, ${ }^{25}$

$$
\begin{aligned}
\int \mathcal{D} \theta_{i} \exp & {\left[-\int \mathrm{d} x_{0} \sum_{i} \frac{A}{T_{0}}\left(\frac{\mathrm{d} \theta_{i}\left(x_{0}\right)}{\mathrm{d} x_{0}}\right)^{2}\right] } \\
= & \int \mathcal{D} \theta_{i} \mathcal{D} \Pi_{i} \\
& \times \exp \left[-\int \mathrm{d} x_{0} \sum_{i}\left\{\frac{T_{0}}{4 A} \Pi_{i}^{2}-i \Pi_{i} \frac{\mathrm{d} \theta_{i}}{\mathrm{~d} x_{0}}\right\}\right],
\end{aligned}
$$

the partition function for the two-dimensional Hamiltonian of eq. (1) is rewritten as

$$
\begin{aligned}
Z= & \int \mathcal{D} \theta_{i}, \mathcal{D} \Pi_{i}, \\
\times & \exp \left[-\int \mathrm{d} x_{0} \sum_{i}\left\{\frac{T_{0}}{4 A} \Pi_{i}^{2}-i \Pi_{i} \frac{\mathrm{d} \theta_{i}}{\mathrm{~d} x_{0}}\right.\right. \\
& \left.-\frac{W_{c}}{T_{0}} \cos 4 \theta_{i}-\frac{W_{d}}{T_{0}} \sin 2 \theta_{i}\right] . \\
& \left.\left.+\frac{J_{0}}{T_{0}} \cos \left(\theta_{i}-\theta_{i+1}+\mu\right)\right\}\right] .
\end{aligned}
$$

Next, we consider the one-dimensional quantum Hamiltonian with ( $\Delta x$ being the lattice constant) given by

$$
\begin{aligned}
\mathcal{H}_{q}= & \sum_{j} \Delta x\left[\frac{T_{0}}{4 A} \Pi_{j}^{2}\right. \\
& -\frac{W_{c}}{T_{0}} \cos 4 \theta_{j}-\frac{W_{d}}{T_{0}} \sin 2 \theta_{j} \\
& -\frac{g_{1 / 4}}{2 \pi^{2} \alpha^{2}} \int \mathrm{d} x \cos 4 \theta_{+}-\frac{g_{1 / 2}}{2 \pi^{2} \alpha^{2}} \int \mathrm{d} x \sin 2 \theta_{+} \\
+ & \left.\frac{J_{0}}{T_{0}} \cos \left(\theta_{j}-\theta_{j+1}+\mu\right)\right], \\
& +\sum_{i} \int \mathrm{d} x_{0} J_{0} \cos \left(\theta_{i}\left(x_{0}\right)-\theta_{i+1}\left(x_{0}\right)+\delta\right),
\end{aligned}
$$


where $\left[\theta_{j}, \Pi_{k}\right]=i \delta_{j k}$ and $j$ denotes the lattice index along $x$-direction. Starting with eq. (A.3), the partition function for the Hamiltonian of eq. $(\mathrm{A} \cdot 3)$ is ${ }^{25}$

$$
\begin{aligned}
Z= & \operatorname{Tr}\left(e^{-\beta \mathcal{H}_{q}}\right) \\
= & \lim _{N \rightarrow \infty} \operatorname{Tr}\left(e^{-\frac{\beta}{N} \mathcal{H}_{q}}\right)^{N} \\
= & \prod_{i} \sum_{\theta_{i}\left(\tau_{0}\right)}\left\langle\theta_{i}\left(\tau_{0}\right)\left|e^{-\Delta \tau \mathcal{H}_{q}} \prod_{j} \sum_{\theta_{j}\left(\tau_{1}\right)}\right| \theta_{j}\left(\tau_{1}\right)\right\rangle\left\langle\theta_{j}\left(\tau_{1}\right)\right| \\
& \times e^{-\Delta \tau \mathcal{H}_{q}} \ldots\left|\theta_{i}\left(\tau_{0}\right)\right\rangle \\
= & \int \mathcal{D} \theta_{j} \mathcal{D} \Pi_{j} \\
& \times \exp \left[-\int_{0}^{\beta} \mathrm{d} \tau \sum_{j} \Delta x\right. \\
& \left\{\frac{T_{0}}{4 A} \Pi_{j}^{2}(\tau)-i \Pi_{j}(\tau) \frac{\mathrm{d} \theta_{j}(\tau)}{\mathrm{d} \tau}\right. \\
- & \frac{W_{c}}{T_{0}} \cos 4 \theta_{j}(\tau)-\frac{W_{d}}{T_{0}} \sin 2 \theta_{j}(\tau) \\
+ & \left.\left.\frac{J_{0}}{T_{0}} \cos \left(\theta_{j}(\tau)-\theta_{j+1}(\tau)+\mu\right)\right\}\right],
\end{aligned}
$$

where $\Delta \tau=\beta / N$ and $\tau$ is the imaginary time. Thus, the present classical Hamiltonian of eq. (1) is equivalent to the quantum Hamiltonian of eq. (A.3). By taking the continuum limit of $\mathcal{H}_{q}$ along $x$-direction, namely $\theta_{j}(\tau) \rightarrow \theta_{+}(\tau, x)$ and $\sum_{j} \rightarrow \int \mathrm{d} x / \Delta x$ with $x=j \Delta x$, one obtains following effective Hamiltonian,

$$
\begin{aligned}
\mathcal{H} & =\frac{v_{\rho}}{4 \pi} \int \mathrm{d} x\left[\frac{1}{K_{\rho}}\left(\partial_{x} \theta_{+}-\nu\right)^{2}+K_{\rho}\left(\partial_{x} \theta_{-}\right)^{2}\right] \\
& +\frac{g_{1 / 4}}{2 \pi^{2} \alpha^{2}} \int \mathrm{d} x \cos 4 \theta_{+}-\frac{g_{1 / 2}}{2 \pi^{2} \alpha^{2}} \int \mathrm{d} x \sin 2 \theta_{+}, \\
& -\frac{g_{v}}{2 \pi^{2} \alpha^{2}} \int \mathrm{d} x \cos \theta_{-},
\end{aligned}
$$

with the commutation relation, $\left[\theta_{+}(x), \partial_{x} \theta_{-}\left(x^{\prime}\right)\right]=-2 \pi i \delta\left(x-x^{\prime}\right)^{23}$ and $K_{\rho}=$ $T_{0} /\left(2 \pi \sqrt{2 A\left|J_{0}\right|}\right), \quad \nu=-\mu / \Delta x, \quad v_{\rho}=\sqrt{\left|J_{0}\right| / 2 A}, g_{1 / 4}=-2(\pi \alpha)^{2} W_{c} / T_{0}$ and $g_{1 / 2}=$ $2(\pi \alpha)^{2} W_{d} / T_{0}$. The Hamiltonian eq. (A.5) has been examined in the 1-D interacting quarterfilled electron system with dimerization. ${ }^{22}$

We comment on the interaction of the vortex in terms of $\theta_{-}(x)$. In eq. (A·3), the Hamiltonian is invariant under the transformation given by $\theta_{i} \rightarrow \theta_{i}+2 \pi$. The excitation expressing such a spatial variation corresponds to the short range topological excitation which is related to the vortex excitation. Actually, in continuum model, such an excitation is expressed as follows. Using the commutation relation, the vortex excitation is generated by unitary trans- 
formation, in terms of $V(x)=e^{i \theta_{-}(x)}$, that is $V\left(x^{\prime}\right) \theta_{+}(x) V\left(x^{\prime}\right)^{\dagger}=e^{i \theta_{-}\left(x^{\prime}\right)} \theta_{+}(x) e^{-i \theta_{-}\left(x^{\prime}\right)}=$ $\theta_{+}(x)+\pi \operatorname{sgn}\left(x-x^{\prime}\right)$. Then taking into account the symmetry of eq. (A.3), one can add the potential, $\int \mathrm{d} x W_{v} \cos \theta_{-}(x)$ to eq. (A.5), since the term is invariant under the unitary transformation representing the lowest vortex excitation ( the vortex potential which has lowest scaling dimension). ${ }^{24}$ Note that the correspondence between classical system and quantum system is given by $x \leftrightarrow \tau, y \leftrightarrow x, b \leftrightarrow \Delta x, \theta \leftrightarrow \theta_{+}, 2 \pi K \phi \leftrightarrow \theta_{-}, K \leftrightarrow 1 / 2 \pi K_{\rho}$ and $\beta \leftrightarrow v_{\rho}$. Thus we obtain the effective classical Hamiltonian eq. (3) which includes the vortex excitation. However, the microscopic calculation is needed to obtain the coefficient, $W_{v}$. In the present paper, we put $W_{v}=\exp \left(-\pi^{2} K / 2\right)$, which is the extrapolation deduced from the isotopic stiffness case. ${ }^{6}$ 


\section{References}

1) V. L. Pokrovsky and A. L. Talapov: Phys. Rev. Lett. 42 (1978) 65, Zh. Eksp. Thoe. Fiz. 78 (1980) 269.

2) B. Horovitz, T. Bohr, J. M. Kosterlitz and H. J. Schulz: Phys. Rev. B 28 (1983) 6596.

3) M. Tsuchiizu, P. Donohue, Y. Suzumura and T. Giamarchi: Eur. Phys. J. B 19 (2001) 185.

4) T. Itakura and Y. Suzumura: to be published in J. Phys. Soc. Jpn. 70 (2001) No.7.

5) J. M. Kosterlitz and D. J. Thouless: J. Phys. C 6 (1973) 1181.

6) J. V. José, L. P. Kadanoff, S. Kirkpatrick and D. R. Nelson: Phys. Rev. B 16 (1977) 1217.

7) D. Boyanovsky: J. Phys. A 22 (1989) 2601.

8) F. D. M. Haldane, P. Bak and T. Bohr: Phys. Rev. B 28 (1983) 2743.

9) H. J. Schulz: Phys. Rev. B 28 (1983) 2746.

10) T. Giamarchi and H. J. Schulz: J. Phys. France 49 (1988) 819.

11) D. Jérome and H.J. Schulz: Adv. Phys. 31 (1982) 299.

12) T. Ishiguro, K. Yamaji and G. Saito: Organic Superconductors (Springer-Verlag, Berlin, 1998).

13) K. Bechgaard and D. Jérome: Phys. Scr. T 39 (1991) 37.

14) J. Moser, M. Gabay, P. Auban-Senzier, D. Jérome, K. Bechgaard and J. M. Fabre: Eur. Phys. J. B 1 (1998) 39.

15) K. Yamaji: J. Phys. Soc. Jpn. 51 (1982) 2787.

16) L. Ducasse, M. Abderrabba, J. Hoarau, M. Pesquer, B. Gallois and J. Gaultier: J. Phys. C 19 (1986) 3805.

17) H. Seo and H. Fukuyama: J. Phys. Soc. Jpn. 66 (1997) 1249.

18) Y. Suzumura: J. Phys. Soc. Jpn. 66 (1997) 3244.

19) T. Itakura and Y. Suzumura: J. Phys. Chem. Solids. 62 (2001) 423.

20) T. Itakura and Y. Suzumura: J. Phys. Soc. Jpn. 68 (1999) 2395.

21) M. Tsuchiizu: Ph.D. thesis (Nagoya University 2001).

22) M. Tsuchiizu, H. Yoshioka and Y. Suzumura: J. Phys. Soc. Jpn. 70 (2001) No. 6 (condmat/0103530).

23) Y. Suzumura: Prog. Theor. Phys. 61 (1979) 1.

24) E. Orignac: unpublished.

25) J. W. Negele and H. Orland: Quantum Many-Particle System (Addison Wesly Pub., Reading, MA, 1998).

26) T. Takahashi, T. Harada, Y. Kobayashi, K. Kanoda, K. Suzuki, K. Murata and G. Saito: Synth. Metals 41-43 (1991) 3985. 